\title{
Quantum fluctuations in the image of a Bose gas
}

\author{
Antonio Negretti ${ }^{1}$ * Carsten Henkel ${ }^{2}$, and Klaus Mølmer ${ }^{1}$ \\ 1. Lundbeck Foundation Theoretical Center for Quantum System Research \\ Department of Physics and Astronomy, University of Aarhus, 8000 Aarhus C, Denmark \\ 2. Institut für Physik und Astronomie, Universität Potsdam, \\ Karl-Liebknecht-Str. 24-25, 14476 Potsdam, Germany
}

\begin{abstract}
We analyze the information content of density profiles for an ultracold Bose gas of atoms and extract resolution limits for observables contained in these images. Our starting point is density correlations that we compute within the Bogoliubov approximation, taking into account quantum and thermal fluctuations beyond mean-field theory. This provides an approximate way to construct the joint counting statistics of atoms in an array of pixels covering the gas. We derive the Fisher information of an image and the associated Cramér-Rao sensitivity bound for measuring observables contained in the image. We elaborate on our recent study on position measurements of a dark soliton [Negretti et al., Phys. Rev. A 77, 043606 (2008)] where a sensitivity scaling with the atomic density as $n^{-3 / 4}$ was found. We discuss here a wider class of soliton solutions and present a detailed analysis of the Bogoliubov excitations and the gapless (Goldstone) excitation modes. These fluctuations around the mean field contribute to the noise in the image, and we show how they can actually improve the ability to locate the position of the soliton.
\end{abstract}

PACS numbers: 03.75.Nt, 06.20.Dk, 37.25.+k

\section{INTRODUCTION}

de Broglie waves of massive particles are very sensitive to perturbations and may serve as efficient probes for electromagnetic fields 1], earth's rotation [2], Casimir forces [3] (or in general to detect weak forces [4]), and particle properties as, for example, the refraction index of a buffer gas [5] or the electric polarizability of an atom [6]. In interferometry, disturbance of the phase of light or matter waves in one arm of the interferometer can be measured by a displacement of the interference fringes with a sensitivity determined by the fringe wavelength and the signal-to-noise ratio (SNR). The SNR is under many circumstances given by the standard shot noise, leading to a resolution that scales with $1 / \sqrt{N}$, where $N$ is the number of detected atoms.

The shot noise limit, however, can be beaten with entanglement and squeezing [7], proving that it is not a fundamental limit and in the scenario of Ref. [8], for example, a particular entangled preparation scheme has been shown to give a phase error scaling as $1 / N^{3 / 4}$, while in principle the Heisenberg limit should provide the optimum sensitivity with a phase error scaling as $1 / N$ [9]. Many analyses of nonclassical metrology with quantum objects have dealt with the situation of particles or fields that may be prepared in suitably entangled states, and entangled measurements may be used after they have experienced the interaction of interest [10]. Conversely, entanglement created by many-body interactions improves the precision of estimating the corresponding coupling constant, as discussed recently [11, 12].

In this paper we arrive at a $1 / N^{3 / 4}$ scaling for a dis-

*E-mail: negretti@phys.au.dk placement measurement with particles that are, however, not entangled at all. We consider a system of bosonic atoms, which are cooled to Bose-Einstein degeneracy and prepared in a joint collective quantum state, described by mean field theory, i.e., by a Hartree-Fock product state wave function. We take for the latter a dark soliton, a topological excitation stabilized by atom-atom interactions. The soliton has a density minimum at a location $q$, and this position can be used to monitor the phase acquired in an atomic matter wave interferometer in the nonlinear regime [13, 14, 15]. A recent experiment has demonstrated the relevance of the relative phase in splitting and recombining a Bose-Einstein condensate (BEC), although the direct observation of solitons was not possible [16]. In a similar way, very recent experiments have generated solitons that oscillate and collide in a harmonic trap, in the crossover regime between one dimension (1D) and three dimensions (3D) [17]. As we have shown in a recent publication [18], the decrease of the soliton width with the number of atoms (a nonlinear effect) gives rise to an improved position resolution, surpassing the shot noise threshold, when more and more particles are used for the experiment.

Atom clouds are typically analyzed by taking (pixelized) absorption or phase contrast images, giving access to the atomic density profile. If one wants to measure some quantity of interest, the resolution is limited by the fluctuations in the image due to counting noise on every pixel. We characterize these fluctuations and specify how to extract, in an (almost) optimal way, information from the noisy data. It turns out that the quantum fluctuations beyond the Hartree-Fock product state actually do not spoil the image resolution, but even improve it slightly, although the power law scaling with the atomic density $\left(\sim n^{-3 / 4}\right)$ is unchanged. In this paper, we present a description of these elements of the theory, providing 
a number of technical ingredients and details that were only briefly mentioned in Ref. [18].

In Sec II, we introduce the quantum image of an atomic cloud, define the associated Fisher information (FI) and recall its connection to optimal parameter estimation, starting from the image data. We treat solitons and vortices in a mean field description with Poissonian counting statistics, and we introduce a general Gaussian Ansatz for the counting statistics within an image, for which the Fisher information can be determined analytically. Different scalings with the atomic density are identified that range between the shot-noise and the Heisenberg limits, depending on the system dimension and the kind of nonlinearity. In Sec III, a quantum field theory of atomic density correlations is developed within the Bogoliubov approximation. We provide a discussion of the role of phonon and zero (or Goldstone) modes, and we present detailed calculations for the density fluctuations in the image of a dark soliton. The location of the soliton is, due to the breaking of translational symmetry of the problem, itself associated with a Goldstone mode, and the corresponding contribution to particle fluctuations are analyzed. In this section, we also construct a nearly optimal protocol for image processing. In Sec IV we give a brief summary and conclusion.

\section{MEAN FIELD IMAGES}

\section{A. Atom density statistics}

The continuous popularity of cold quantum gas physics is due, in part, to the possibility of measuring atomic density profiles by fluorescence or absorption imaging. Adopting the language of second quantization, the corresponding observable is the intensity or density operator,

$$
\hat{n}(\mathbf{x})=\hat{\Psi}^{\dagger}(\mathbf{x}) \hat{\Psi}(\mathbf{x}),
$$

integrated along a line of sight. (We shall omit this integral in the following.) An 'image' thus corresponds to a set of measured densities $\{\rho(\mathbf{x}) \mid \mathbf{x}\}$. Averaging over many images, one gets an estimate for the expectation value $\bar{\rho}(\mathbf{x})=\langle\hat{n}(\mathbf{x})\rangle$. If the detector integrates the density signal over some small, but finite area $\mathcal{A}_{\mathrm{px}}(s)$ (the 'pixel' no. $s=1,2, \ldots M)$, one deals with a discrete set of operators like

$$
\hat{n}_{s}=\int_{\mathcal{A}_{\mathrm{px}}(s)} \mathrm{d} \mathbf{x} \hat{n}(\mathbf{x}) .
$$

Correlations between the atomic density in different points are related to the field operator in the following way, using the bosonic commutation relations

$$
\begin{aligned}
\left\langle\hat{n}(\mathbf{x}) \hat{n}\left(\mathbf{x}^{\prime}\right)\right\rangle= & \left\langle\hat{\Psi}^{\dagger}(\mathbf{x}) \hat{\Psi}^{\dagger}\left(\mathbf{x}^{\prime}\right) \hat{\Psi}\left(\mathbf{x}^{\prime}\right) \hat{\Psi}(\mathbf{x})\right\rangle \\
& +\langle\hat{n}(\mathbf{x})\rangle \delta\left(\mathbf{x}-\mathbf{x}^{\prime}\right) .
\end{aligned}
$$

This quantity defines the density correlation function

$$
\mathcal{P}\left(\mathbf{x}, \mathbf{x}^{\prime}\right)=\left\langle\hat{n}(\mathbf{x}) \hat{n}\left(\mathbf{x}^{\prime}\right)\right\rangle-\langle\hat{n}(\mathbf{x})\rangle\left\langle\hat{n}\left(\mathbf{x}^{\prime}\right)\right\rangle
$$

that will play a key role in this paper.

Let us illustrate these concepts for the case that the quantized field operator $\hat{\Psi}(\mathbf{x})$ can be reduced to a single mode. This is a common approximation at low temperatures where a macroscopic fraction of atoms condenses into a single spatial wave function. The operator $\hat{\Psi}(\mathbf{x})$ is then replaced by a single annihilation operator $\hat{a}_{0}$ multiplying a classical complex field $\Phi(\mathbf{x})$, and the average density is given by

$$
\langle\hat{n}(\mathbf{x})\rangle=N_{0}|\Phi(\mathbf{x})|^{2},
$$

where $N_{0}=\left\langle\hat{a}_{0}^{\dagger} \hat{a}_{0}\right\rangle$ is the number of atoms in the 'condensate mode' $\Phi(\mathbf{x})$ (itself normalized to unity). The density correlations, essentially the structure factor of the system [19], are found as

$$
\begin{aligned}
\mathcal{P}\left(\mathbf{x}, \mathbf{x}^{\prime}\right)= & \left(\Delta N_{0}^{2}-N_{0}\right)|\Phi(\mathbf{x})|^{2}\left|\Phi\left(\mathbf{x}^{\prime}\right)\right|^{2} \\
& +N_{0}|\Phi(\mathbf{x})|^{2} \delta\left(\mathbf{x}-\mathbf{x}^{\prime}\right) .
\end{aligned}
$$

The first term, proportional to the Mandel parameter [20], vanishes if the system is in an eigenstate of the operator $\hat{a}_{0}$, i.e., a coherent state (Poisson statistics). The second term describes local fluctuations at the same position (for an image: in the same pixel), with a variance that is equal to the mean density (the mean atom number on the pixel).

It is intuitively clear that when more than a single spatial mode are taken into account in the field operator expansion, valuable information about the field's quantum state is hidden in the correlations of the atomic density [21]. This has been discussed recently for the Mott insulator-superfluid transition [22, 23], and exploited in measurements on the strongly correlated Mott insulator phase with ultracold ${ }^{87} \mathrm{Rb}$ atoms released from an optical lattice 24]. Excited modes also introduce additional fluctuations into an image, however. The competition between these two effects will be the central theme of this paper.

\section{B. Parameter estimation}

\section{Information measures}

The full joint probability distribution of the atom numbers in every pixel provides the complete 'counting statistics' of an image. The information content of an image is given by the counting statistics via the classical information entropy $I$, which for a pixelized image is given by (e.g., see [25])

$$
I=-\sum_{\rho_{1}, \ldots \rho_{M}} p\left(\left\{\rho_{s}\right\}\right) \log p\left(\left\{\rho_{s}\right\}\right) .
$$


In (7), $p\left(\left\{\rho_{s}\right\}\right)$ is the joint probability measure for the occurrence of detection events with $\rho_{s}$ atoms detected in pixel $\operatorname{px}(s)$ ( $M$ is the total number of pixels).

A particular application of this information concept appears when we want to estimate a parameter $q$ "hidden" in the image, i.e., the counting statistics is a function $p\left(\left\{\rho_{s}\right\} ; q\right)$ of the parameter $q$, referred to as the likelihood function (LF) in statistical estimation theory. The hidden parameter can be the fraction of particles in the condensate mode, the width of the cloud, or the position of a "solitonic" excitation, i.e., the location of a minimum in the density profile. This will be our example throughout the analysis. Information processing theory provides an explicit formula for the optimal signal-tonoise ratio in measuring $q$. It can be translated into a lower limit on the variance, the Cramér-Rao bound (CRB), $\operatorname{Var}(q) \geq 1 / F(q)$. Here, the Fisher information $F(q)$ is given by $[25,26]$

$$
F(q)=-\sum_{\rho_{1}, \ldots \rho_{M}} p\left(\left\{\rho_{s}\right\} ; q\right) \frac{\partial^{2} \log p\left(\left\{\rho_{s}\right\} ; q\right)}{\partial q^{2}} .
$$

In a quantum mechanical framework, the FI has a geometrical interpretation as distance (metric), depending on a parameter $q$, in the space of density operators 27]. The multiple sum in Eq.(8) is difficult to evaluate in general, and we shall focus in this paper on two schemes where the calculations are feasible: $(i)$ the single-mode approximation for the field operator, assuming Poissonian atom number counting statistics; (ii) a Gaussian approximation for the probability measure, where the results can be expressed in terms of the average density and the density correlations. The latter scheme will be applied to a multi-mode field theory within the Bogoliubov approximation.

To estimate the parameter $q$ we require a definite prescription of how to extract it from the data that fluctuate from shot to shot. We shall in particular identify an optimal prescription that permits to saturate the CramérRao bound.

\section{Poissonian counting statistics}

The single-mode approximation is well known as the mean-field theory for BEC. We assume here that the variance of condensate particles is normal, $\Delta N_{0}^{2}=N_{0}$ (Poisson statistics) so that the density correlations are given by the last term in Eq.(6). The mean field theory is equivalent to a Hartree product state Ansatz for the many-body wave function. As a result, the probability measure $p\left(\left\{\rho_{s}\right\}\right)$ factorizes into Poissonian statistics for each pixel. The summation in Eq. (8) can then be performed analytically. When we take the limit of infinitely small pixels, one finds an integral over the spatial coordinate of the image [28]

$$
F(q)=4 \int \mathrm{d} x\left[\frac{\partial|\Phi(x ; q)|}{\partial q}\right]^{2},
$$

where we have made the dependence of the average density profile $|\Phi(x ; q)|^{2}$ on the parameter $q$ explicit. (From here on, the complex field $\Phi$ is not normalized to unit norm, but its square gives the mean density of the condensate particles.)

The meaning of this formula can be illustrated by a discussion of the optimal signal processing strategy. Given the image data $\rho(x ; q)$, we construct, as in Refs. 28, 29], a linear filter $g(x)$ to provide an estimate for $q$ :

$$
\begin{aligned}
S(q) & =\int \mathrm{d} x g(x) \rho(x ; q) \\
& \approx q \int \mathrm{d} x g(x) \partial_{q} \rho(x ; 0),
\end{aligned}
$$

where $g(x)$ is a local gain function on the pixel at position $x$ that can take positive and negative values. In the second step we have assumed, without loss of generality, that the signal vanishes when the parameter $q$ is zero, and we have performed a Taylor expansion of the density profile, $\rho(x ; q) \simeq \rho(x ; 0)+q \partial_{q} \rho(x ; 0)$. The expectation value of the signal, $\bar{S}(q)$, is simply obtained in terms of $\bar{\rho}(x ; q)=\langle\hat{n}(x)\rangle$ and its derivative.

The variance of the signal is found by squaring Eq. (10) (first line) and expressing the average in terms of the density correlation function (4)

$$
\Delta S^{2}=\int \mathrm{d} x \mathrm{~d} y g(x) g(y) \mathcal{P}(x, y ; q) .
$$

Recalling that different pixels are uncorrelated [Eq.(6) reduces to its last term], this variance reduces to

$$
\Delta S^{2}=\int \mathrm{d} x g^{2}(x) \bar{\rho}(x ; 0) .
$$

We can now choose the gain function $g(x)$ such that the signal-to-noise ratio SNR $=\bar{S}^{2}(q) / \Delta S^{2}$ is maximized. This optimization problem has the following solution, as pointed out in Ref.[29] for a coherent state of light populating a single spatial mode:

$$
g_{\text {opt }}(x)=\frac{\alpha}{|\Phi(x ; 0)|}\left(\frac{\partial|\Phi(x ; q)|}{\partial q}\right)_{q=0} .
$$

Here $\alpha$ is a normalization constant. This gain function can also be interpreted as an optimized spatial mode (the "noise mode" in the language of Ref.[29]), onto which Eq.(10) projects the image. The minimum uncertainty $\Delta q$ for the parameter estimation corresponds to an SNR of unity, and this reaches the Cramér-Rao bound $(\Delta q)^{2} \equiv \operatorname{Var}(q)=1 / F(q)$ with $F(q)$ given by Eq.(9). If the wave function $\Phi$ were proportional to $\sqrt{N_{0}}$, we would find a shot-noise limited resolution, $\Delta q \propto 1 / N_{0}^{1 / 2}$. Due to atom-atom interactions, this limit can be overcome, as we shall see.

\section{Gaussian images}

Given the mean atom number per pixel $\bar{\rho}_{s}(q)$ and the (pixelized) density correlation matrix $P_{s j}(q)$ with 
[cf. Eq.(4)]

$$
\begin{aligned}
\bar{\rho}_{s}(q) & =\int_{\mathcal{A}_{\mathrm{px}}(s)} \mathrm{d} x \rho(x ; q), \\
P_{s j}(q) & =\int_{\mathcal{A}_{\mathrm{px}}(s)} \mathrm{d} x \int_{\mathcal{A}_{\mathrm{px}}(j)} \mathrm{d} y \mathcal{P}(x, y ; q),
\end{aligned}
$$

we may make the assumption of a joint Gaussian probability distribution. Since a Gaussian is fully characterized by its first and second moments the probability measure is simply given by [25]

$p(\boldsymbol{\rho} ; q)=\frac{(2 \pi)^{-M / 2}}{\sqrt{\operatorname{det}(\mathbf{P})}} \exp \left[-\frac{1}{2}(\boldsymbol{\rho}-\overline{\boldsymbol{\rho}}) \cdot \mathbf{P}^{-1}(\boldsymbol{\rho}-\overline{\boldsymbol{\rho}})\right]$,

where the vector $\boldsymbol{\rho}$ of length $M$ collects the detected atom number variables, $\overline{\boldsymbol{\rho}}$ collects the corresponding mean values, and $\mathbf{P}$ is the covariance matrix with elements $P_{s j}(q)$. The likelihood function $p(\boldsymbol{\rho} ; q)$ depends on $q$ through $\bar{\rho}_{s}(q)$ and $P_{s j}(q)$.

With the Ansatz (16) for the LF and by replacing the sum over discrete particle counts by continuous integrals, the FI is given by the following analytical expression [18

$$
\begin{aligned}
F(q)= & \frac{1}{2}\left\{\frac{\partial_{q}^{2} \operatorname{det}(\mathbf{P})}{\operatorname{det}(\mathbf{P})}-\left[\frac{\partial_{q} \operatorname{det}(\mathbf{P})}{\operatorname{det}(\mathbf{P})}\right]^{2}\right. \\
& \left.+\sum_{s, j}\left[\frac{\partial^{2}\left(\mathbf{P}^{-1}\right)_{s j}}{\partial q^{2}} P_{s j}+2\left(\mathbf{P}^{-1}\right)_{s j} \frac{\partial \bar{\rho}_{s}}{\partial q} \frac{\partial \bar{\rho}_{j}}{\partial q}\right]\right\} .
\end{aligned}
$$

If no correlations exist between neighboring pixels, this expression reduces to Eq. (9) for the Poisson case, provided one takes both the limit of small pixel size and large average atom number per pixel. This is as expected since for a large average, Poisson and Gaussian statistics become similar. We discuss an example taken from Ref. 18] below.

Correlations between pixels may increase or decrease the FI and make the parameter estimate more or less precise. A detailed calculation is discussed in Sec IIC.

\section{Examples: kinks and vortices}

We evaluate now the Fisher information for measurements of the position of a solitonic excitation in a Bosecondensed gas. We consider first a $1 \mathrm{D}$ setting where the quantum field theory is given by the following nonlinear evolution equation for the field operator [the GrossPitaevskii equation (GPE)] [19, 30]

$$
i \hbar \frac{\partial}{\partial t} \hat{\Psi}=\left[-\frac{\hbar^{2}}{2 m} \frac{\partial^{2}}{\partial x^{2}}+V_{\text {ext }}+g \hat{\Psi}^{\dagger} \hat{\Psi}\right] \hat{\Psi},
$$

where $m$ is the atom mass, $V_{\text {ext }}$ is an external potential, and $g$ the binary interaction strength proportional to the $s$-wave scattering length $a_{s}$. Within mean-field theory, the field operator is replaced by the complex order parameter $\Phi(x)$ (the condensate wave function or order parameter) that also satisfies Eq.(18). The mean atom density is then $\bar{\rho}(x)=|\Phi(x)|^{2}$, thus normalizing the order parameter to the particle density.

A family of "dark" solitonic excitations of the Bose condensate exists for repulsive interactions $(g>0)$. In the homogeneous case $\left(V_{\text {ext }}=0\right)$, it is given by [19]

$$
\Phi(x ; q)=\frac{\sqrt{n}}{c}\left\{i v+\sqrt{c^{2}-v^{2}} \tanh [\kappa(v)(x-q)]\right\},
$$

which shows a local minimum in the density at the position $q \equiv q(t)=q(0)+v t$. The width of this dip is specified by $\kappa(v)=(\sqrt{2} \xi)^{-1} \sqrt{1-v^{2} / c^{2}}$ where $\xi=\hbar / \sqrt{2 m g n}$ is the so-called healing length and $n$ is the one-dimensional "background" density. The soliton moves with constant velocity $v$ that does not exceed the sound velocity $c=\sqrt{n g / m}$. For a soliton at rest, the density is strictly zero at $x=q$, and the phase of the condensate wave function increases by $\pi$ when crossing this point (a "kink soliton"). The kink becomes wider and the density dip disappears as the velocity $v \rightarrow \pm c$. The wavefunction in (19) is normalised such that the atom density asymptotically approaches the constant value of $n$ (actually, after few healing lengths). Such solitons have been created in BEC experiments by shining a laser field on one half of the atomic ultracold cloud, which induced a relative phase by the AC Stark effect [31].

Topological excitations in two dimensions are vortices where the phase of the wave function increases by a multiple of $2 \pi$ when circling around a zero in the density. We discuss an example below.

\section{Dark soliton}

Due to the shot noise fluctuations in the detected atoms the density dip is washed out and one has to find a good estimate for the kink position $q$. The formula (9) for the FI within Poisson statistics can be evaluated exactly for the dark soliton given by Eq.(19). The result is [recall that Eq.(91) implies the limit of infinitely small pixels]

$$
\begin{aligned}
F= & \frac{4 n \sqrt{2}}{\xi} \frac{v}{c}\left\{\arctan \left[\frac{v / c-c /(2 v)}{\sqrt{2} \xi \kappa(v)}\right]\right. \\
& \left.-\arctan \left[\frac{v / c}{\sqrt{2} \xi \kappa(v)}\right]\right\}+\frac{8 n}{3}\left(2+\frac{v^{2}}{c^{2}}\right) \kappa(v) .
\end{aligned}
$$

As expected from translation invariance, after integration over $x$, this does not depend on the soliton position $q$. In Fig $\left[1\right.$ we plot the minimum uncertainty $\Delta q=F(q)^{-1 / 2}$ of the soliton position in units of the condensate healing length, as a function of the soliton velocity $v$. This behaviour makes good sense: when the soliton is almost at rest, the dip is very sharp and a precise knowledge of 


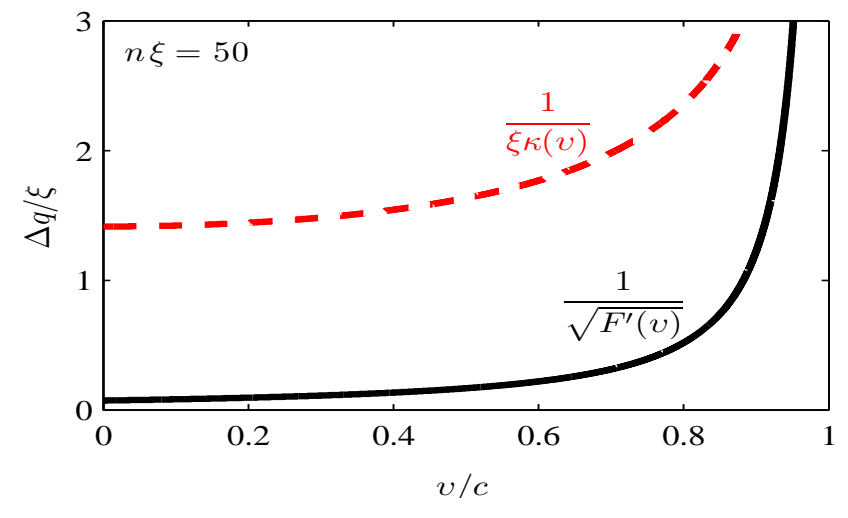

Figure 1: (Colour online) Soliton position uncertainty $\Delta q$, in units of the healing length $\xi$, versus soliton velocity $v$ ( $c$ is the speed of sound). The scaled Fisher information is given by $F^{\prime}=F \xi^{2}$.

the soliton position can be gathered; when the velocity approaches $c$, the dip in the density becomes very shallow, and less information is available from density measurements. For comparison, the dashed curve shows the width $1 / \kappa(v)$ of the density dip: we see that the measurement precision can largely exceed this value. The maximum information is

$$
v=0: \quad F=\frac{16}{3 \sqrt{2}} \frac{n}{\xi}=\frac{16}{3} \frac{\sqrt{m g}}{\hbar} n^{3 / 2},
$$

which implies that, for a soliton at rest, the uncertainty in $q$ scales like $n^{-3 / 4}$ with the background density $n$. As pointed out in Ref. [18], this is a better scaling than the usual shot noise limit $\left(\sim n^{-1 / 2}\right)$. We emphasize that this enhancement does not require any squeezed or otherwise entangled multi-atom state. It simply follows from the shorter wavelengths that occur in a BEC matter wave interferometer due to the atom-atom interactions.

The scaling with the density can be understood with the following statistical argument: the atom number on a pixel of area $\Delta x$ at position $x$ is given by $N(x)=$ $\rho(x) \Delta x \pm \sqrt{\rho(x) \Delta x}$, assuming fluctuations at the shotnoise level. From error propagation theory the uncertainty $\Delta q$ on the soliton position $q$ is given by

$$
\Delta q=\frac{\Delta N(x)}{|\mathrm{d} \rho(x) / \mathrm{d} x| \Delta x} \sim \frac{\xi}{\sqrt{n \Delta x}},
$$

where the healing length $\xi$ sets the scale for the density variation around the dip. By processing different data points across the relevant region, this uncertainty can be reduced by a factor $1 / \sqrt{M}$ where $M \approx \xi / \Delta x$ is the number of pixels across the dip, which leads to a precision of the order of

$$
\Delta q \sim \sqrt{\frac{\xi}{n}} \propto n^{-3 / 4} .
$$

Let us now turn to the Gaussian approximation to the likelihood function of an image and discuss the Fisher
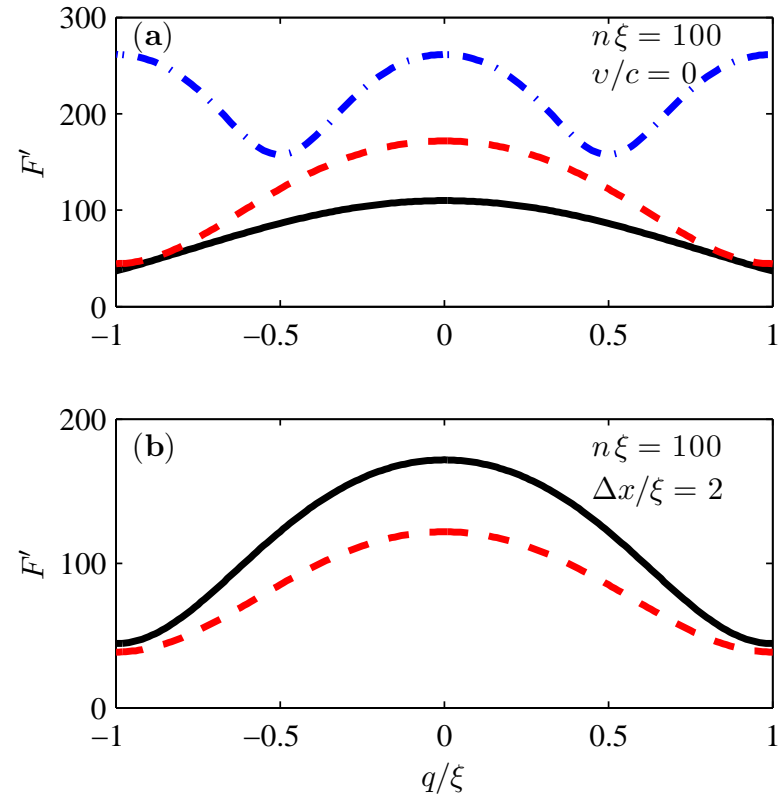

Figure 2: (Colour online) (a) Rescaled Fisher information as a function of soliton position, $q$, for a Poissonian LF. The solid (black) line is the result for pixel size $\Delta x / \xi=3$, the dashed (red) line for $\Delta x / \xi=2$, and the dashdot (blue) for $\Delta x / \xi=1$. (b) Fisher information as a function of soliton position, $q$, for a Poissonian LF, shown for two different soliton velocities and fixed $\Delta x$. The solid (black) line is for $v=0$ and the dashed (red) line is for $v=0.3 c$.

information (17) for the dark soliton. We recover the variance and mean of the Poisson distribution by choosing a covariance matrix with $P_{s j}=\operatorname{Var}\left(\rho_{s}\right) \delta_{s j}=\bar{\rho}_{s} \delta_{s j}$, with the variance of the number of atoms on the $s$-th pixel equal to its mean value $\bar{\rho}_{s}(q)$.

The integral in Eq.(14) with the order parameter given in (19) can be worked out analytically and the derivative with respect to $q$ performed. The FI (17), scaled to $F^{\prime}=$ $F \xi^{2}$, then becomes

$$
F^{\prime}=n^{2} \xi^{2}\left(1-v^{2} / c^{2}\right)^{2}\left[\sum_{s} \frac{g_{s}}{\bar{\rho}_{s}(0)}+\frac{1}{2} \sum_{s} \frac{g_{s}}{\bar{\rho}_{s}^{2}(0)}\right],
$$

where we have put $q=0$ without loss of generality and

$$
\begin{aligned}
& g_{s}=\left\{\operatorname{sech}^{2}\left[\kappa(v)\left(x_{s}+\Delta x\right)\right]-\operatorname{sech}^{2}\left[\kappa(v) x_{s}\right]\right\}^{2}, \\
& \bar{\rho}_{s}(0)=n \Delta x-2 n \xi^{2} \kappa(v)\left\{\tanh \left[\kappa(v)\left(x_{s}+\Delta x\right)\right]\right.
\end{aligned}
$$

with $x_{s}=s \Delta x$, and $s$ an integer. This expression is giving those pixels a stronger weight where the density profile significantly changes, as could have been expected. As a consequence, pixels at the outer limits where the atomic density is flat (already a few healing lengths away 
from the soliton position $q$ ) do not contribute to the FI. We can therefore safely assume that the soliton is located well in the interior of a detection window for imaging, and even take the limit of an infinitely large window. (For a discussion of the limit $\Delta x \rightarrow 0$, see Ref.[18].) That is also confirmed in Fig 2(a): we show the dependence of the FI on the soliton position $q$ within a pixel. The oscillations occur at the pixel size $\Delta x$, but for $\Delta x \rightarrow 0$, their amplitude becomes smaller and $F^{\prime}$ is almost flat. In Fig 2(b), we fix the value of $\Delta x$ and change the soliton velocity $v$. Also in that case the FI has a maximum for $q=0$ (soliton at the border between two pixels).

\section{Trapped soliton}

The previous discussion shows that the information content is concentrated near the soliton position. This suggests that trapped solitons, in an inhomogeneous background, behave in a similar way.

Consider first a soliton at rest in a square potential of length $2 \ell$. Assuming a single-mode picture with Poissonian number statistics, the FI becomes

$$
F^{\prime}=n^{2} \xi^{2} \sum_{s} \frac{g_{s}(\Delta x)}{\bar{\rho}_{s}(0)} \vartheta\left(\ell-\left|x_{s}\right|\right),
$$

where the step function $\vartheta(x)$ appears. As expected, only those pixels covering the confining box contribute to the information. In the limit $\ell \rightarrow \infty$, we recover the result of an infinite Bose gas given by the first sum of (24) with $v=0$. The same discussion can be applied to the Gaussian approximation for the complete counting statistics.

In a harmonic trap, the order parameter can be approximately written as 32 ]

$$
\Phi(x ; q) \simeq \Phi_{\mathrm{bg}}(x) \tanh \left(\frac{x-q}{\sqrt{2} \xi_{0}}\right),
$$

where $\Phi_{\mathrm{bg}}(x)$ is the Thomas-Fermi (TF) solution for the trap ground state 33], $\xi_{0}$ is the healing length for the background density at the soliton position (it depends on the density $\left.n_{0}=\left|\Phi_{\mathrm{bg}}(q)\right|^{2}[34]\right)$. Let us focus on solitons at rest, close to the centre of the trap, $q \approx 0$, and much smaller than the size $R_{\mathrm{TF}}$ of the background field. The FI (9) then turns out to be

$$
F_{\text {trap }}(q) \simeq \frac{16}{3 \sqrt{2}} \frac{n_{0}}{\xi_{0}}
$$

which is very similar to the homogeneous case, keeping the density $n_{0}$ the same.

This result can be written in different ways by expressing the central density $n_{0}$ in terms of other parameters. Taking, for example, the total number of atoms, we find

$$
F_{\text {trap }} \simeq 14.1 \frac{N_{0}}{a_{x}^{2}},
$$

where $a_{x}$ is the (single-particle) ground state size in the harmonic trap. This leads to the usual shot-noise scaling $N_{0}^{-1 / 2}$ for the soliton position. Most of the atoms, however, are not needed for the image since the information content is concentrated near the soliton minimum. In this respect, the trapped situation is favorable compared to the homogeneous case since the central density is higher and the healing length is smaller in the center than in the condensate wings. This can be made quantitative by comparing the harmonic trap to a homogeneous sample of the same size and same total number of atoms. The ratio of the Fisher Information between the two cases is then

$$
\frac{F_{\text {hom }}}{F_{\text {trap }}} \simeq 0.11,
$$

where corrections of order $\xi / R_{\mathrm{TF}}$ are neglected, consistent with the TF approximation. It should be noted that the size of the system in both cases scales in a different way with the atom number.

\section{Solitons with three-body interactions}

The GPE relies on the two-body pseudopotential $g \delta(\mathbf{x})$, which describes the interaction between the particles. One can also consider low-dimensional Bose superfluids in a strong-coupling regime with three-body collisions. We analyze here the solitonic solution in a quasi 1D setup governed by the equation (homogeneous case)

$$
i \hbar \frac{\partial}{\partial t} \Phi=\left[-\frac{\hbar^{2}}{2 m} \frac{\partial^{2}}{\partial x^{2}}+\gamma|\Phi|^{4}\right] \Phi
$$

with the universal coupling constant $\gamma=(\hbar \pi)^{2} /(2 m)$. This system has been investigated in detail in Refs. 35, 36]. There exists an analytical soliton solution for the order parameter $\Phi(x ; q)=\sqrt{n} f(x) e^{i \phi(x)}$, with

$$
\begin{aligned}
f^{2}(x ; q) & =1-\frac{3\left[1-(v / c)^{2}\right]}{2+\sqrt{1+3(v / c)^{2}} \cosh \left[\kappa^{\prime}(x-q)\right]} \\
\kappa^{\prime} & =2 \pi n \sqrt{1-(v / c)^{2}} .
\end{aligned}
$$

This kind of solitons can also appear in a degenerate electron plasma [37], since the equation for the order parameter describing the electron density is almost the same. As opposed to Eq.(19), the soliton width $1 / \kappa$ is scaling here $\sim 1 / n$ with the density $n$, and we therefore expect an improvement in the soliton kink estimation. Indeed, using the Poisson formula (9) for the Fisher information we obtain for a soliton at rest

$$
F=2 \sqrt{3} \pi \ln (2+\sqrt{3}) n^{2}
$$

that can be compared to Eq.(21). With such topological excitations we thus reach a $1 / n$ scaling as in the Heisenberg limit, without any entanglement or squeezing. 


\section{Vortex line}

Finally, we consider a two-dimensional situation with a vortex line. The vortex solution of the 3D GPE is described by a core region where the density goes to zero. Vortices are not stable solutions, however, and only in a frame rotating with a high angular velocity they correspond to local or global energy minima [19]. Vortices in a rotating $\mathrm{BEC}$ with respect to the lab frame were observed in several experiments, as for example in Ref. 38].

We assume the simple case of a Bose gas with uniform confinement along the $z$ axis (length $L$ ) and homogeneous in the $x y$-plane. The solution of the GPE for a gas rotating around the $z$ axis is given by [19]

$$
\Phi(\mathbf{r} ; q)=\sqrt{n / L} f(x, y, z ; q)\left(\frac{x-q+\mathrm{i} y}{x-q-\mathrm{i} y}\right)^{s / 2}
$$

where $n$ is the two-dimensional density, $f$ is a real function, $(q, 0)$ is the position of the vortex line in the $x y$ plane, and $s$ is an integer (the 'winding number' or 'topological charge'). The order parameter (36) is an eigenfunction of the angular momentum with eigenvalue $\hbar s$. Since we are interested in the calculation of the FI (9), which depends only on the absolute value $|\Phi|$, the angular dependence is not relevant in the present setting. In the following we specialise to the case of a singly charged vortex $s= \pm 1$. The function $f$ can then be well approximated by 30 ]

$$
f(x, y, z ; q) \simeq \frac{\sqrt{(x-q)^{2}+y^{2}}}{\sqrt{2 \xi^{2}+(x-q)^{2}+y^{2}}} .
$$

We generalize Eq. (9) for the FI to this two-dimensional setting and obtain

$$
F=4 \int_{0}^{L} \mathrm{~d} z \int \mathrm{d} x \mathrm{~d} y\left[\frac{\partial|\Phi(r, \varphi, z ; 0)|}{\partial q}\right]^{2}=\pi n
$$

where the integration is easy to perform in cylindrical coordinates. It is interesting to note that for a vortex line, the FI depends on the (two-dimensional) density $n$ linearly. Hence, the fact that the vortex core scales with the healing length does not improve the shot-noise limit for its position detection. This can be understood from the simple argument of Eq.(23) because the relevant number of atoms scales with $n \xi^{2} \sim \hbar^{2} /\left(2 m g_{2 D}\right)$ which is only weakly dependent on the density: the dimensionality of the topological excitation plays a crucial role.

\section{Dimensionality crossover}

The last example has shown that the dimension of the topological excitation is very important. Coming back to the quasi-1D situation, we discuss here briefly the crossover of the nonlinear terms in the GPE as one changes the confinement transverse to the long trap axis. The 1D solitons described by Eq.(18) are difficult to realize in an experiment because one needs a high ratio between radial and axial trap frequency. When transverse effects are taken into account with more accuracy, the GPE is modified into a $1 \mathrm{D}$ non-polynomial Schrödinger equation [39, 40, 41, 42]. This changes, for example, the oscillation frequency of a dark soliton in a harmonic trap [41]. In the limits of weak and strong coupling, the nonlinearity in the effective GPE becomes polynomial, and analytical results for solitonic solutions (bright and dark) can be given [39]. By inspecting these dark solitons, it is easy to show that the Fisher information scales, in the strong coupling regime, as $n^{2}$ with the background density $n$, identical to the scaling found in SecIC3. This beats the shot noise scaling $(F \propto n)$ and illustrates that also with the help of two-body interactions, one can reach the scaling of the Heisenberg limit in a strongly interacting system.

\section{QUANTUM FIELD THEORY FOR IMAGES}

\section{A. Motivation}

Mean field theories are ubiquitous in physics and play an important role in the explanation of many phenomena in condensed matter physics [43, 44], e.g. superconductivity in metals, and even in high energy physics as for example the quark condensate in the so called instanton ensemble in QCD (Quantum Chromodynamics) [45]. The mean field approach describes successfully key features of quantum degenerate dilute gases [19, 30], for example the static, dynamic, and thermodynamic properties of trapped Bose-Einstein condensates [46], and it has been well confirmed in a number of experiments (see, e.g., Ref.[47]). The mean field approximation applied to an ultracold Bose gas leads to the Gross-Pitaevski equation (18). The stationary solutions of the GPE represent the macroscopically occupied spatial mode functions when the temperature of the trapped gas is well below the critical transition temperature. This concept can be formally translated, in the $U(1)$ symmetry broken approach, in the following way: the total matter field is split into $\hat{\Psi}(x)=\Phi(x)+\delta \hat{\Psi}(x)$, where $\delta \hat{\Psi}(x)$ represents quantum fluctuations around the Gross-Pitaevskii solution $\Phi(x)$. The mean field approach essentially neglects the fluctuation field.

Mean field methods, however, assess only the average atomic density and do not provide information about quantum noise correlations, which are important in the understanding of quantum phase transitions in ultracold atoms, e.g., anti-ferromagnetic structures or charge density waves [48]. 


\section{B. Bogoliubov approximation}

\section{Phonon and zero modes}

Even at zero temperature, the particle density shows fluctuations that lead to the phenomenon of "quantum depletion" [19, 49, 50]. This can be described by linearizing the total many-body problem around the mean field solution. In other words, one expands the second quantised Hamiltonian up to quadratic terms in the fluctuation field $\delta \hat{\Psi}$. The dynamics of the latter is then generated by the Bogoliubov-de Gennes linear operator

$$
\mathcal{L}=\left(\begin{array}{cc}
\mathcal{H}+g|\Phi|^{2} & g \Phi^{2} \\
-g \Phi^{* 2} & -\mathcal{H}-g|\Phi|^{2}
\end{array}\right)
$$

where we have introduced the Gross-Pitaevskii Hamiltonian

$$
\mathcal{H}=-\frac{\hbar^{2}}{2 m} \frac{\partial^{2}}{\partial x^{2}}+V_{\text {ext }}(x)+g|\Phi(x)|^{2}-\mu,
$$

and $\mu$ is the chemical potential.

The mode expansion of the fluctuation operator $\delta \hat{\Psi}$ is complicated by the fact that $\mathcal{L}$ has eigenvectors with eigenvalue zero. These arise from continuous symmetries that leave invariant the energy of the Bose-Einstein order parameter $\Phi$ [43]. A well-known example is the $U(1)$ global phase invariance of the GPE (40), another one is the translation in space of the soliton solution (19) for a homogeneous system. By choosing a dark soliton solution with a definite phase $\theta$ and a given position $q$, one has spontaneously broken these symmetries. According to the non-relativistic Goldstone theorem [44, 51], this spontaneous symmetry breaking is associated with gapless excitation modes, the Goldstone modes, for any system of particles with finite range interactions. The Goldstone modes produce quantum fluctuations of the phase and position of the soliton order parameter, even at zero temperature. The global phase is conjugate to the number of particles, and the phase fluctuations can indeed be interpreted as a consequence of the variations of the chemical potential with particle number [33].

The eigenvalues $E_{k}$ of the Bogoliubov-de Gennes operator (39) correspond to eigenvectors $\left(u_{k}, v_{k}\right)$ that we denote "phonon modes". They are normalized and orthogonal according to

$$
\delta_{k p}=\int \mathrm{d} x\left\{u_{k}^{*}(x) u_{p}(x)-v_{k}^{*}(x) v_{p}(x)\right\}
$$

and are accompanied by partner modes $\left(v_{k}{ }^{*}, u_{k}{ }^{*}\right)$ with negative norm and eigenvalue $-E_{k}^{*}$. This construction fails for the so-called zero modes $\left(u_{\alpha}, v_{\alpha}\right)$ that are in the kernel of $\mathcal{L}$ [50, 52, 53]. For the global $U(1)$ symmetry, the zero mode is $\left(\Phi,-\Phi^{*}\right)$ that is generated by applying $-i \partial / \partial \theta$ to $\left(\Phi e^{i \theta}, \Phi^{*} e^{-i \theta}\right)$. The norm (41) of this mode is zero. We need partner or adjoint modes $\left(u_{\alpha}^{\text {ad }}, v_{\alpha}^{\text {ad }}\right)$ to saturate the completeness relation in the space of fluctuation fields. They can be constructed by solving the equation $\mathcal{L}\left(u_{\alpha}^{\mathrm{ad}}, v_{\alpha}^{\mathrm{ad}}\right)=m_{\alpha}\left(u_{\alpha}, v_{\alpha}\right)$. The generalized eigenvalue $m_{\alpha}$ is called the "effective mass". One gets zero modes of $\mathcal{L}^{2}$ and a completeness relation in the form [53]

$$
\begin{aligned}
\delta(x-y)= & \sum_{k}\left\{u_{k}(x) u_{k}^{*}(y)-v_{k}^{*}(x) v_{k}(y)\right\} \\
& +\sum_{\alpha}\left\{u_{\alpha}(x)\left[u_{\alpha}^{\mathrm{ad}}(y)\right]^{*}-v_{\alpha}^{\mathrm{ad}}(x) v_{\alpha}^{*}(y)\right\}
\end{aligned}
$$

where $\alpha$ enumerates all broken symmetries and the adjoint modes are normalized such that

$$
\delta_{\alpha \beta}=\int \mathrm{d} x\left\{u_{\alpha}^{\mathrm{ad} *}(x) u_{\beta}(x)-v_{\alpha}^{\mathrm{ad} *}(x) v_{\beta}(x)\right\} .
$$

In our one-dimensional soliton system, we expect two Goldstone modes associated with the global soliton phase $\theta$ and its position $q$. The corresponding quantum fluctuations will prove important for our analysis. We give the phonon and zero modes for this geometry in Appendix A.

The fluctuation operator $\delta \hat{\Psi}(x)$ around the GrossPitaevskii solution $\Phi(x)$ is expanded as

$$
\begin{aligned}
\delta \hat{\Psi}(x)= & \sum_{k}\left\{\hat{b}_{k} u_{k}(x)+\hat{b}_{k}^{\dagger} v_{k}^{*}(x)\right\} \\
& +\sum_{\alpha}\left\{\hat{P}_{\alpha} u_{\alpha}^{\mathrm{ad}}(x)-i \hat{Q}_{\alpha} u_{\alpha}(x)\right\}
\end{aligned}
$$

The operators are constructed to implement the commutation relation $\left[\delta \hat{\Psi}(x), \delta \hat{\Psi}^{\dagger}(y)\right]=\delta(x-y): \hat{b}_{k}, \hat{b}_{k}^{\dagger}$ are bosonic annihilation and creation operators, $\left[\hat{b}_{k}, \hat{b}_{p}^{\dagger}\right]=$ $\delta_{k p}$, and for all broken symmetries in the problem, the "position" and "momentum" operators $\hat{Q}_{\alpha}, \hat{P}_{\alpha}$ are canonically conjugate, $\left[\hat{Q}_{\alpha}, \hat{P}_{\beta}\right]=i \delta_{\alpha \beta}$. The completeness and orthogonality relations (42, 43) are compatible with the construction

$$
\begin{aligned}
& \hat{Q}_{\alpha}=i \int \mathrm{d} x\left\{u_{\alpha}^{\mathrm{ad} *}(x) \delta \hat{\Psi}(x)-v_{\alpha}^{\mathrm{ad} *}(x) \delta \hat{\Psi}^{\dagger}(x)\right\} \\
& \hat{P}_{\alpha}=\int \mathrm{d} x\left\{\delta \hat{\Psi}^{\dagger}(x) u_{\alpha}(x)-\delta \hat{\Psi}(x) v_{\alpha}(x)\right\}
\end{aligned}
$$

The operator $\hat{P}_{\alpha}$ can be identified with the generator of the symmetry transformation behind the corresponding zero mode.

\section{Mean density and correlations}

We now provide a general framework for the mean atomic density and the density correlations within Bogoliubov theory. Going beyond Ref.[18], we consider the case of phonon modes at finite temperature. The field expansion (44) is useful for the computation of expectation values because different modes are not correlated. 
Evaluating the average density, we get

$$
\begin{aligned}
\left\langle\hat{\Psi}^{\dagger}(x) \hat{\Psi}(x)\right\rangle= & |\Phi(x)|^{2}+\sum_{k}\left(1+\left\langle\hat{b}_{k}^{\dagger} \hat{b}_{k}\right\rangle\right)\left|v_{k}(x, q)\right|^{2} \\
& +\sum_{k}\left\langle\hat{b}_{k}^{\dagger} \hat{b}_{k}\right\rangle\left|u_{k}(x, q)\right|^{2}+\mathcal{Z}(x),
\end{aligned}
$$

with the occupation number $\left\langle\hat{b}_{k}^{\dagger} \hat{b}_{k}\right\rangle=1 /\left(e^{\beta E_{k}}-1\right)$. For thermal states, $\left\langle\hat{b}_{q}\right\rangle=0$. The so-called quantum depletion is related to the finite contribution $\sim\left|v_{k}\right|^{2}$ of phonon modes even at zero temperature: this is a direct manifestation of quantum density fluctuations. Finite temperature adds thermal contributions to the phonon modes.

The zero mode contribution is obtained as

$$
\begin{gathered}
\mathcal{Z}(x)=\sum_{\alpha}\left\{\left|u_{\alpha}^{\mathrm{ad}}(x)\right|^{2}\left\langle\hat{P}_{\alpha}^{2}\right\rangle+\left|u_{\alpha}(x)\right|^{2}\left\langle\hat{Q}_{\alpha}^{2}\right\rangle\right. \\
\left.-\operatorname{Re}\left[u_{\alpha}^{*}(x) u_{\alpha}^{\mathrm{ad}}(x)\right]-\operatorname{Im}\left[u_{\alpha}^{*}(x) u_{\alpha}^{\mathrm{ad}}(x)\right]\left\langle\left\{\hat{P}_{\alpha}, \hat{Q}_{\alpha}\right\}\right\rangle\right\},
\end{gathered}
$$

where $\{\cdot, \cdot\}$ denotes the anticommutator. For the global $U(1)$ symmetry, the relevant operator averages are: $\left\langle\hat{P}_{\theta}^{2}\right\rangle=N_{0},\left\langle\hat{Q}_{\theta}^{2}\right\rangle=1 /\left(4 N_{0}\right)$, and $\left\langle\left\{\hat{P}_{\theta}, \hat{Q}_{\theta}\right\}\right\rangle=0$, they follow from the assumption that the condensate mode is in a coherent state with $N_{0}$ particles on average. The state for the Goldstone mode associated with soliton displacement is discussed in Sec IIIB3 below. For the moment, we only assumed that the operators $\hat{Q}_{\alpha}, \hat{P}_{\alpha}$ average to zero which is plausible since they appear only quadratically in the Hamiltonian.

When quantum fluctuations around the mean field are taken into account, different spatial locations can become correlated beyond the level of Eq. ([6) because they probe the same delocalized excitation modes. We find the density correlation function $\mathcal{P}(x, y ; q)$ [Eq.(4)] by a straightforward expansion of the four-point field correlations to second order in the fluctuation operator $\delta \hat{\Psi}$ (this is consistent with the Bogoliubov approximation). Thus we get for phonons in a thermal state

$$
\begin{aligned}
\mathcal{P}(x, y ; q) \simeq & \left\langle\left[\Phi^{*}(x) \delta \Psi(x)+\delta \Psi^{\dagger}(x) \Phi(x)\right]\right. \\
& \left.\times\left[\Phi^{*}(y) \delta \Psi(y)+\delta \Psi^{\dagger}(y) \Phi(y)\right]\right\rangle \\
= & \sum_{k}\left\{\left(1+\left\langle\hat{b}_{k}^{\dagger} \hat{b}_{k}\right\rangle\right) f_{k}(x) f_{k}^{\prime}(y)\right. \\
& \left.+\left\langle\hat{b}_{k}^{\dagger} \hat{b}_{k}\right\rangle f_{k}(y) f_{k}^{\prime}(x)\right\} \\
& +\sum_{\alpha}\left\{\left\langle\hat{P}_{\alpha}^{2}\right\rangle \eta_{\alpha}(x) \eta_{\alpha}^{*}(y)+\left\langle\hat{Q}_{\alpha}^{2}\right\rangle \varphi_{\alpha}(x) \varphi_{\alpha}^{*}(y)\right\},
\end{aligned}
$$

where we introduced the functions

$$
\begin{aligned}
& f_{k}(x)=\Phi^{*}(x) u_{k}(x)+v_{k}(x) \Phi(x) \\
& f_{k}^{\prime}(x)=\Phi^{*}(x) v_{k}^{*}(x)+u_{k}^{*}(x) \Phi(x)
\end{aligned}
$$

for the phonons, and the abbreviations

$$
\begin{aligned}
& \eta_{\alpha}(x)=\Phi^{*}(x) u_{\alpha}^{\mathrm{ad}}(x)+u_{\alpha}^{\mathrm{ad} *}(x) \Phi(x) \\
& \varphi_{\alpha}(x)=i \Phi^{*}(x) u_{\alpha}(x)-i u_{\alpha}^{*}(x) \Phi(x)
\end{aligned}
$$

that appear like "mode functions" for the zero mode operators. We have simplified Eq. (49) by noting that $\operatorname{Im}\left[u_{\alpha}^{*}(x) u_{\alpha}^{\text {ad }}(x)\right]=0$ for the zero mode functions given in Appendix A. To proceed, we have to specify the quantum state of the soliton displacement modes.

\section{Quantum statistics of soliton position}

In the second-quantized many-body theory, the soliton position is described by an operator $\hat{Q}_{q}$ whose fluctuations "fill" the density dip, as discussed by Dziarmaga [32, 54]. In a homogeneous geometry, the dynamics of $\hat{Q}_{q}$ is similar to a free particle, the corresponding Hamiltonian in the Bogoliubov approximation being $\hat{P}_{q}^{2} / 2 m_{q}$. The stationary states of this Hamiltonian are momentum eigenstates where the soliton position is maximally uncertain. However, the effective mass occurring here is negative, $m_{q}=-4 m(n / \kappa)$. This mimicks the negative kinetic energy of the classical (non-quantized) moving soliton [55, 56]. A similar phenomenon occurs in a harmonic trap where the soliton displacement mode has a negative frequency (the mode is called anomalous) [32]. These degrees of freedom are therefore thermodynamically unstable, and we cannot use thermal statistics. Dziarmaga in Ref. 32] has suggested an alternative approach to specify the quantum state of this degree of freedom, to be used as initial condition for the subsequent dynamics. The idea is to "pin" the soliton to the position $q$ by minimizing the density $\hat{\Psi}^{\dagger}(q) \hat{\Psi}(q)$, which is a quadratic form in $\hat{P}_{q}$ and $\hat{Q}_{q}$ [see Eq. (48) at $x=q$ ]. As shown in Ref. [18], one finds in this way a Gaussian state similar to the ground state of a harmonic oscillator with respect to the "Hamiltonian" (in quotes since it has not the dimensions of energy):

$$
\hat{h}\left(\hat{P}_{q}, \hat{Q}_{q}\right)=\frac{1}{16 n} \hat{P}_{q}^{2}+n \kappa^{2} \hat{Q}_{q}^{2}-\frac{\kappa}{4},
$$

where the mode functions of Appendix A, Eqs. A7 A8 have been used. The ground state of this Hamiltonian gives $\langle\hat{h}\rangle=0$ and can also be written as the vacuum state corresponding to the annihilation operator

$$
\hat{b}_{q}=\frac{-i}{\sqrt{8 n \kappa}} \hat{P}_{q}-\sqrt{2 n \kappa} \hat{Q}_{q}
$$

With the help of $\hat{b}_{q}$ and its conjugate operator, we can write the expansion of $\delta \hat{\Psi}(x)$ in the same form as for the phonon modes.

We consider here two classes of states that generalize this ground state: "squeezed" states and "thermal" states. The squeezed state depends on the positive parameter $\zeta$ : it is defined as a Gaussian state with quadrature variances

$$
\left\langle\hat{P}_{q}^{2}\right\rangle_{\zeta}=\frac{2 n \kappa}{\zeta}, \quad\left\langle\hat{Q}_{q}^{2}\right\rangle_{\zeta}=\frac{\zeta}{8 n \kappa},
$$




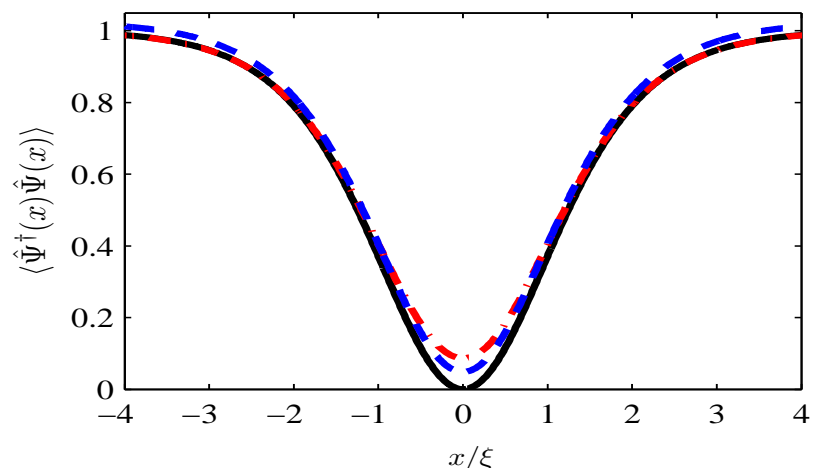

Figure 3: (Colour online) Density profiles in the notch: the solid (black) line corresponds to the squeezing parameter $\zeta=$ 1 whereas the dashdot (red) to $\zeta=100$, while the dashed (blue) line corresponds to the thermal state with parameter $\tau=5 / \xi$. Other parameters are: $\ell=10 \xi, n \xi=100$, and 140 phonon modes have been taken into account.

where $\zeta=1$ corresponds to the ground state. For this state, we get an average density $\langle\hat{h}\rangle_{\zeta}=$ $(\kappa / 8)\left(\zeta^{-1}+\zeta-2\right) \geq 0$. The density at $x=q$ vanishes for $\zeta=1$, and Fig 3 actually shows the result of Eq.(47), including contributions from quantum noise, for that case.

The thermal state is defined by analogy to the canonical ensemble as a state that maximizes entropy at a given mean value of $\hat{h}$. This mean value is given by $\langle\hat{h}\rangle_{\tau}=\frac{1}{2} \kappa /(\exp [\kappa /(2 \tau)]-1)$ and it is controlled by a parameter $\tau$ with the dimension density. The quadratures are

$$
\left\langle\hat{P}_{q}^{2}\right\rangle_{\tau}=2 n \kappa \operatorname{coth}[\kappa /(4 \tau)], \quad\left\langle\hat{Q}_{q}^{2}\right\rangle_{\tau}=\frac{\operatorname{coth}[\kappa /(4 \tau)]}{8 n \kappa} .
$$

We note that these states give average density profiles that resemble a partially filled dark soliton, as illustrated in Fig 3. The deviations from the "optimal case" are controlled by the parameters $\zeta$ or $\tau$. The quantum states constructed here are not stationary states of the Bogoliubov Hamiltonian, however, and will evolve in time, as discussed in Ref.[32].

\section{Discussion of results}

In Fig 3 we show density profiles corresponding to different values of the squeezing parameter $\zeta=1,100$ and for the thermal parameter $\tau=5 / \xi$. The plot confirms that the choice $\zeta=1$ (or when $\tau \rightarrow 0^{+}$for a "thermal" $q$-state) gives the state with minimum depletion that best resembles a condensate with a perfect soliton. For $\tau=5 / \xi$ the minimum in the notch is not zero (as for $\zeta \neq 1$ ), but the density at the edges becomes higher than the density of the squeezed quantum state, reflecting the "thermal" and the long wave nature of the zero $q$-mode state.

For what concerns the density correlations, it is convenient to bring them in a form where the $\delta$-correlated term in Eq.(3) that appears due to normal ordering, is subtracted. To this effect, we use the completeness relation (42) to rewrite the sum over $u_{k}(x) u_{k}^{*}(y)$ in the correlation function (49) as

$$
\mathcal{P}(x, y)=\Phi(x) \Phi(y)[\delta(x-y)+\mathcal{J}(x, y)]
$$

where the function $\mathcal{J}(x, y)$ is found as

$$
\begin{aligned}
\mathcal{J}(x, y)= & \operatorname{Re}\left\{\sum_{k}\left[2 v_{k}(x) v_{k}^{*}(y)+u_{k}(x) v_{k}^{*}(y)+v_{k}(x) u_{k}^{*}(y)\right]\right\} \\
& +4\left\{\left\langle\hat{P}_{\theta}^{2}\right\rangle u_{\theta}^{\mathrm{ad}}(x) u_{\theta}^{\mathrm{ad} *}(y)+\left\langle\hat{Q}_{q}^{2}\right\rangle u_{q}(x) u_{q}^{*}(y)\right\}-\sum_{\alpha}\left\{u_{\alpha}(x)\left[u_{\alpha}^{\mathrm{ad}}(y)\right]^{*}+u_{\alpha}^{\mathrm{ad}}(x) u_{\alpha}^{*}(y)\right\} .
\end{aligned}
$$

In Fig 4 we show the behavior of $\mathcal{J}(x, y)$ for two different values of the squeezing parameter. Between $\zeta=1$ (left) and $\zeta=100$ (right), the overall magnitude of $\mathcal{J}(x, y)$ changes significantly, demonstrating a strong influence of the soliton displacement mode. For a large uncertainty in $\hat{Q}_{q}(\zeta=100)$, the function $\mathcal{J}(x, y)$ is positive everywhere and concentrated near $x \approx y \approx q$. The choice $\zeta=1$ leads to negative values (anticorrelations) along the diagonal $x=y$ away from the point $x=y=q$.

The calculation of the Fisher information is done nu- merically by using the phonon and zero modes of Appendix A normalizing them in a box of length $2 \ell$. In Fig 5 (a), we show the Fisher information for different approximations: a single-mode BEC with Poisson statistics [dotted line: homogeneous system, solid line: in a finite box, Eq.227] is compared to a multi-mode calculation with a Gaussian counting statistics (dashed, dot-dashed, and thin solid lines). The correlation matrix gives contributions to the FI via the derivatives of $\mathbf{P}^{-\mathbf{1}}$ and $\operatorname{det}(\mathbf{P})$ with respect to the soliton position [see Eq.(17)]. We 

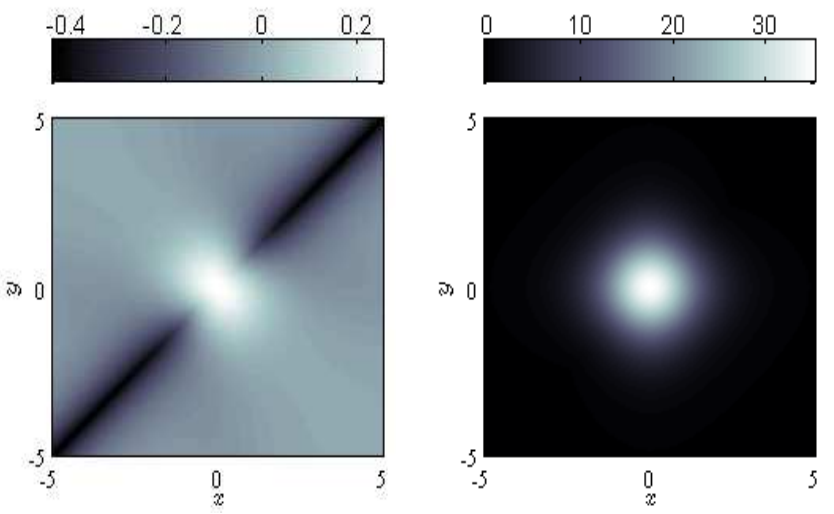

Figure 4: Plot of the function $\mathcal{J}(x, y)$ defined in Eq.(57). Parameters: $\ell=10 \xi, n \xi=100$, and 140 phonon modes have been taken into account. On the left, squeezing parameter $\zeta=1$ (optimal) and on the right $\zeta=100$.

have chosen the 'optimal dark soliton' $\zeta=1.0$ (dashed line) and a slightly filled (squeezed) one, $\zeta=1.5$ (dashdotted line). The thin solid line corresponds to the FI for a thermal state with a parameter $\tau=0.2 / \xi$.

First, it is interesting to note that one gets more information in a box than in the homogeneous case. This is a finite-size effect which enhances the impact of "missing atoms" in the soliton center. Second, the exact result for the Gaussian multi-mode theory (17) shows that correlations, including the zero modes, increase the level of information that we can extract, beyond mean-field theory (single-mode approximation). Moreover, the dashed curve for $\zeta=1$ shows that minimizing the quantum "filling" of the notch provides the highest information. The divergence of $F^{\prime}$ at small pixel size is due to the failure of the Gaussian approximation which becomes unphysical, as the average atom counts per pixel drop below unity, see Ref.[18].

In Fig $[$ (b) we show the scaled Fisher information versus the scaled density $n$. Both mean-field and multi-mode theory give a linear scaling with $n$ which translates into the same power law $n^{-3 / 4}$ for the sensitivity of the soliton position.

In order to understand why the inclusion of noise with the Bogoliubov description provides an even better resolution than the mean field, we use the formalism of signal processing introduced in Sec IB 2. The signal-to-noise ratio gives an assessment of the amount of information that can be extracted from a given statistical estimation strategy. By using the completeness relation (42) and the result (57) it can be easily shown that the noise $\Delta S^{2}$ [Eq.(11)] splits into

$$
\Delta S^{2}=\Delta S_{\mathrm{MF}}^{2}+\Delta S_{\mathrm{ph}}^{2}-\Delta S_{\mathrm{G}}^{2}
$$

for any gain function $g(x)$. The three terms here are the contributions of the mean field, the phonon contribution, and the sum over the Goldstone (or zero) modes. The Goldstone contribution is negative and reduces the noise even below the phonon level. This is related to
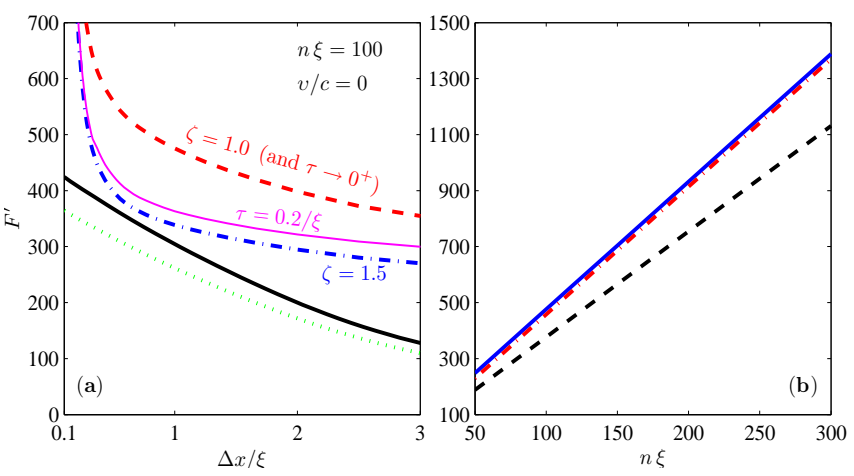

Figure 5: (Colour online) (a) Rescaled Fisher information $F^{\prime}=F \xi^{2}$ versus pixel size for different regimes: solid (black) line for a Poissonian counting statistics in a box and the dotted (green) line in the uniform Bose gas limit. The other lines take into account quantum and thermal density fluctuations, using a Gaussian approximation to the counting statistics. They differ in the quantum state of the Goldstone mode associated with the soliton displacement. Dashed (red): state with zero density at soliton position ("squeezing" parameter $\zeta=1$, effective "temperature" $\tau=0$ ); dashdot (blue): squeezed state $\zeta=1.5$; thin solid (magenta): thermal state $\tau=0.2 / \xi$. (b) Rescaled Fisher information versus linear density: dashed (black) line corresponds to Eq.(21), and the dashdot (red) line is the information extracted from the signal-tonoise ratio for a gain function $\mathbf{g}^{\text {ao }}$ (see text), and the solid (blue) line is the Fisher information within Gaussian and Bogoliubov approximations for a finite pixel size $\Delta x=0.7 \xi$, and $\zeta=1$. In both pictures the numerical simulations were made with a box length $2 \ell=20 \xi$, and by considering 140 phonon modes.

the behaviour of the function $\mathcal{J}(x, y)$ in (57) for $\zeta=1$, Fig 4(left): its negative values are larger in magnitude that its positive ones. Recall that for the density correlations (56), $\mathcal{J}(x, y)$ is multiplied by the product $\Phi(x) \Phi(y)$ that is zero for $x=y=q$ and increases to a positive constant $n$ for $x=y \neq q$ after a few healing lengths. This 
further enhances the negative contributions of $\mathcal{J}(x, y)$, and reduces the noise $\Delta S^{2}$ relative to the mean-field approach.

The above discussion clarifies how the Cramér-Rao bound can be smaller for multi-mode fields compared to the mean field description. But can one also identify a strategy to reach the CRB? In the Poissonian case, without correlations, the SNR with the optimal gain function $g_{\text {opt }}(x)$ [Eq. [13)] reaches the sensitivity given by the Fisher information (9), and this happens when the variance $\Delta S^{2} \propto \bar{S}(q)$. We shall proceed in the same way with the multi-mode theory, but work with finite pixel areas to avoid the breakdown of the Gaussian approximation. In that case signal and noise are given by

$$
\begin{array}{r}
\bar{S}(q)=\mathbf{g} \cdot \frac{\partial \overline{\boldsymbol{\rho}}}{\partial q} \Delta x, \\
\Delta S^{2}=\mathbf{g} \cdot \mathbf{P} \mathbf{g} \Delta x^{2},
\end{array}
$$

where the vector $\mathbf{g}$ (length $M$ ) represents the values of the gain function $g\left(x_{s}\right)$ on the pixels. These expressions are the discrete versions of Eqs.(10, 111).

We can achieve $\Delta S^{2} \propto \bar{S}(q)$ by solving the linear system $\mathbf{P} \mathbf{g}=\partial_{q} \overline{\boldsymbol{\rho}}$, giving an optimal gain function $\mathbf{g}^{\text {ao }}$. It is then easy to check that the last term in Eq.(17) becomes

$$
\sum_{s, j}\left(\mathbf{P}^{-1}\right)_{s j} \frac{\partial \bar{\rho}_{s}}{\partial q} \frac{\partial \bar{\rho}_{j}}{\partial q}=\frac{\partial \overline{\boldsymbol{\rho}}}{\partial q} \cdot \mathbf{P}^{-1} \frac{\partial \overline{\boldsymbol{\rho}}}{\partial q} \propto \bar{S}(q) .
$$

We were not able to find a similar proportionality for the term $\operatorname{Tr}\left[\left(\partial^{2} \mathbf{P}^{-1} / \partial q^{2}\right) \mathbf{P}\right]$. A numerical analysis, illustrated in Fig 5(b), shows that we are nevertheless very close to the CRB with the gain function $\mathrm{g}^{\text {ao }}$.

We emphasize that this argument only requires the first and second moments of the counting statistics to optimize the SNR. It does not directly rely on the Fisher information which is only available within the approximation that the entire counting statistics is Gaussian.

\section{CONCLUSION}

We have analyzed in this paper the quantum fluctuations in the counting statistics of an atomic density image. We used Bogoliubov theory, and we observed that zero modes, due to the breaking of the $U(1)$ and translation symmetries, makes quantitatively a significant change in the information content of the counting distributions. We applied our theory to identify the ultimate information theoretical limits for position measurements of dark solitons and other topological excitations in a quasi one-dimensional and two-dimensional BoseEinstein condensate.

In the case of a pure condensate, where all particles occupy the same quantum state described by the GPE, the best estimation of the soliton position has an uncertainty that scales, for a weakly interacting system, as $n^{-3 / 4}$ with the linear background density $n$. This beats by a factor $n^{-1 / 4}$ the scaling of the classical shot noise limit $1 / \sqrt{n}$. We emphasize that this limit is reached without the need of any entanglement or squeezing of the system state. Even more favorable scalings are found for strongly interacting systems where the nonlinearity appears with a different exponent in the GPE. In optical and atomic interferometry, shorter wavelengths provide a better resolution of phase changes, and we can explain our result as a consequence of the high wave number content, i.e., the steepness, of the soliton dip. A similar improvement could also be obtained by simply applying fast counterpropagating beams of atoms. We believe, however, that the stability properties of solitons and the fact that there is no or only little net particle current makes this system suitable for interferometric investigations over longer time scales compared to a thermal beam.

We have investigated the influence of quantum fluctuations with the help of Bogoliubov theory in the weakly interacting case. The scaling law $\left(n^{-3 / 4}\right)$ remains stable, but the prefactor is different. The Goldstone modes that are associated to spontaneously broken symmetries, in fact increase the sensitivity of the measurements. While signal processing theory provides a theoretical limit for the sensitivity (the Cramér-Rao bound), it generally does not provide a method to achieve this limit. In our approach, we were able to find a nearly optimal filtering function, and showed with a signal-to-noise ratio analysis that it almost reaches the Cramér-Rao bound.

\section{Acknowledgments}

The authors A.N. and K.M. acknowledge financial support from the European Union Integrated Project SCALA. K.M. acknowledges support of the Multidisciplinary University Research Initiative "Quantum Metrology with Atomic Systems", administered by the Office of Naval Research. C.H. thanks the Deutsche Forschungsgemeinschaft for support (He 2849/3).

\section{Appendix A: EXCITATION MODES FOR A DARK SOLITON}

Here we sketch the derivation of the Bogoliubov eigenmodes for a system with a soliton in a box of length $2 \ell$. The analysis complements results given in Ref. 54]. We are looking for the eigenstates of the Bogoliubovde Gennes operator (39) with $V_{\text {ext }}(x)=0$. The background or condensate field is given by the wave function $\Phi=\Phi(x ; q)$ of Eq. (19). We focus here on the stationary case (soliton velocity $v=0$ ). Simple and compact expressions for the Bogoliubov eigenmodes can be found in the limit $\ell \gg \xi$, when the soliton is located well within the quantization box. The integral of $|\Phi(x ; q)|^{2}$ over the box gives the number of condensed atoms $N_{0}$ :

$$
N_{0}=2 \ell n-2 n / \kappa
$$


where the negative correction describes the atoms "missing" in the soliton notch. We have set $\kappa \equiv \kappa(v=0)=$ $1 / \sqrt{2} \xi$. For the sake of simplicity in the notation, we shall hereafter drop the parametric dependence on $q$ in the order parameter $\Phi$, the phonon modes $u_{k}, v_{k}$, and the zero modes.

\section{Phonon modes}

Following the approach in Ref. 54, 57], we find that the modes of $\mathcal{L}$ in a box with periodic boundary conditions can be written as

$$
\begin{aligned}
& \left.\begin{array}{l}
u_{k}(x) \\
v_{k}(x)
\end{array}\right\}=M_{k} e^{i k x}\left\{\frac{k}{\kappa} \operatorname{sech}^{2}[\kappa(x-q)]\right. \\
& \left.+\beta_{k}^{ \pm}\left(\frac{k}{2 \kappa}+i \tanh [\kappa(x-q)]\right)\right\},
\end{aligned}
$$

where the upper [lower] sign applies to $u_{k}\left[v_{k}\right]$, respectively. Here, $M_{k}$ is a normalisation constant [given in Eq. (A6)],

$$
\beta_{k}^{ \pm}=\left(\frac{k}{\kappa}\right)^{2} \pm \frac{2 E_{k}}{m c^{2}}
$$

$c$ is the speed of sound, and the phonon energy is given by

$$
E_{k}=\hbar c|k| \sqrt{1+\frac{k^{2}}{4 \kappa^{2}}} .
$$

This energy is the same as on a homogeneous background condensate.

The presence of the soliton becomes manifest in the total phase shift of a phonon passing from the left to the right end of the box. It is given by $2 k \ell+\Delta \varphi(k)$, where $\Delta \varphi(k)=2 \arctan (2 \kappa / k)$ is due to the interaction with the density notch. The quantisation condition for the wave number $k$ is thus:

$$
2 k_{j} \ell+\Delta \varphi\left(k_{j}\right)=2 \pi j \quad j= \pm 1, \pm 2, \ldots
$$

[The case $j=0$ is excluded because $|2 k \ell+\Delta \varphi(k)|>\pi$.] In Sec III B, sums over $k$ are understood as running over this discrete set of wavenumbers.

From the normalization condition (41) for the phonon modes, we obtain the normalization constant $M_{k}$

$$
M_{k}=\frac{\kappa}{2 k} \sqrt{\frac{\kappa g n}{2 \epsilon_{k}}}\left\{\ell \kappa\left[\frac{k^{2}}{2 \kappa^{2}}+2\right]-1\right\}^{-1 / 2} .
$$

In Fig 6 we show the first three eigenfunctions $v_{j}$ with $j=1,2,3$, for $q=0$.

\section{Zero modes}

For our quantum degenerate Bose gas the Goldstone modes originate from the breaking of the global $U(1)$

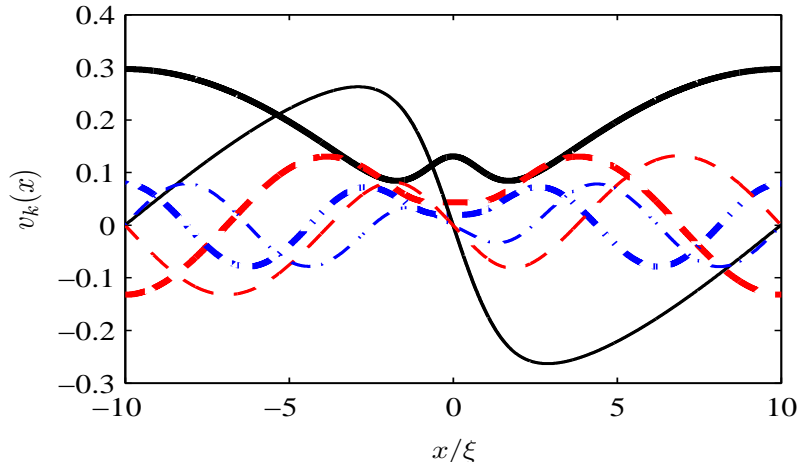

Figure 6: (Colour online) First three Bogoliubov mode functions $v_{k}(x)$ for $q=0$, normalized in a box of length $2 \ell=20 \xi$ (periodic boundary conditions). Thick (thin) lines represent the real (imaginary) part of $v_{k}(x)$, respectively. Solid (black) line: $k_{1}=0.5379(\pi / \ell)$, dashed (red) line: $k_{2}=1.6093(\pi / \ell)$, dashdot (blue) line $k_{3}=2.6704(\pi / \ell)$. These $k$-values can be compared to a free particle where $k_{j}=0,1,2(\pi / \ell)$.

phase symmetry and translational symmetry by assigning a phase $\theta$ and a value of the displacement $q$ to the order parameter $\Phi$ respectively. In order to determine the associated mode functions, we simply differentiate the order parameter $\Phi$ with respect to its global phase $\theta$ or the parameter $q$, as described in Sec IIIB and Ref. 54. We obtain

$$
\begin{aligned}
& u_{\theta}(x)=\Phi(x) \\
& u_{q}(x)=-i \kappa \sqrt{n} \operatorname{sech}^{2}[\kappa(x-q)]
\end{aligned}
$$

with $v_{\alpha}(x)=-u_{\alpha}^{*}(x)$. We have chosen here $\theta=0$, i.e., a real-valued $\Phi(x)$, otherwise the phase of $u_{q}(x)$ would be different. With respect to the Bogoliubov scalar product Eq.(41), the modes (A7) have zero norm and are mutually orthogonal. The zero modes are accompanied by adjoint vectors that satisfy $\mathcal{L} u_{\alpha}^{\text {ad }} \propto u_{\alpha}$ and are found as

$$
\begin{aligned}
u_{\theta}^{\mathrm{ad}}(x) & =\frac{\kappa}{2\left(N_{0} \kappa+n\right)}\left[\Phi(x)+i x u_{q}(x)\right] \\
u_{q}^{\mathrm{ad}}(x) & =\frac{-i}{4 \sqrt{n}} .
\end{aligned}
$$

They have zero norm because $v_{\alpha}^{\text {ad }}(x)=u_{\alpha}^{\text {ad* }}(x)$. The adjoint modes are mutually orthogonal with respect to Eq. (41) which explains the presence of the second term in Eq. $(\mathrm{A} 8)$. For the normalization and this extra term, we consider the limit $\kappa \ell \gg 1$ and neglect exponentially small corrections. 
[1] M. Vengalattore, J. M. Higbie, S. R. Leslie, J. Guzman, L. E. Sadler, and D. M. Stamper-Kurn, Phys. Rev. Lett. 98, 200801 (2007).

[2] S. Stringari, Phys. Rev. Lett. 86, 4725 (2001).

[3] J. M. Obrecht, R. J. Wild, M. Antezza, L. P. Pitaevskii, S. Stringari, and E. A. Cornell, Phys. Rev. Lett. 98, 063201 (2007).

[4] S. G. Bhongale and E. Timmermans, Phys. Rev. Lett. 100, 185301 (2008).

[5] J. Schmiedmayer, M. S. Chapman, C. R. Ekstrom, T. D. Hammond, S. Wehinger, and D. E. Pritchard, Phys. Rev. Lett. 74, 1043 (1995).

[6] C. R. Ekstrom, J. Schmiedmayer, M. S. Chapman, T. D. Hammond, and D. E. Pritchard, Phys. Rev. A 51, 3883 (1995).

[7] V. Giovannetti, S. Lloyd, and L. Maccone, Science 306, 1330 (2004).

[8] L. Pezzè and A. Smerzi, Europhys. Lett. 78, 30004 (2007).

[9] P. Bouyer and M. A. Kasevich, Phys. Rev. A 56, R1083 (1997).

[10] V. Giovannetti, S. Lloyd, and L. Maccone, Phys. Rev. Lett. 96, 010401 (2006).

[11] S. Boixo, A. Datta, S. T. Flammia, A. Shaji, E. Bagan, and C. M. Caves, Phys. Rev. A 77, 012317 (2008).

[12] S. Boixo, A. Datta, M. J. Davis, S. T. Flammia, A. Shaji, and C. M. Caves, Phys. Rev. Lett. 101, 040403 (2008).

[13] T. F. Scott, R. J. Ballagh, and K. Burnett, J. Phys. B 31, L329 (1998).

[14] A. Negretti and C. Henkel, J. Phys. B 37, L385 (2004).

[15] R. G. Scott, T. E. Judd, and T. M. Fromhold, Phys. Rev. Lett. 100, 100402 (2008).

[16] G.-B. Jo, J.-H. Choi, C. A. Christensen, T. A. Pasquini, Y.-R. Lee, W. Ketterle, and D. E. Pritchard, Phys. Rev. Lett. 98, 180401 (2007).

[17] A. Weller, J. Ronzheimer, C. Gross, D. Frantzeskakis, G. Theocharis, P. Kevrekidis, J. Esteve, and M. Oberthaler, arXiv:0803.4352 (2008).

[18] A. Negretti, C. Henkel, and K. Mølmer, Phys. Rev. A 77, 043606 (2008).

[19] L. P. Pitaevskii and S. Stringari, Bose-Einstein Condensation, International Series of Monographs on Physics No. 116 (Oxford University, New York, 2003).

[20] L. Mandel and E. Wolf, Optical coherence and quantum optics (Cambridge University Press, Cambridge, 1995).

[21] E. Altman, E. Demler, and M. D. Lukin, Phys. Rev. A 70, 013603 (2004).

[22] I. B. Mekhov, C. Maschler, and H. Ritsch, Phys. Rev. Lett. 98, 100402 (2007).

[23] I. B. Mekhov, C. Maschler, and H. Ritsch, Nature Physics 3, 319 (2007).

[24] S. Fölling, F. Gerbier, A. Widera, O. Mandel, T. Gericke, and I. Bloch, Nature 434, 481 (2005).

[25] P. Réfrégier, Noise Theory and Application to Physics (Springer, New York, 2004).

[26] H. Cramér, Mathematical Methods of Statistics (Princeton University, Princeton, 1946).

[27] S. L. Braunstein and C. M. Caves, Phys. Rev. Lett. 72, 3439 (1994).

[28] N. Treps, V. Delaubert, A. Maitre, J. M. Courty, and
C. Fabre, Phys. Rev. A 71, 013820 (2005).

[29] V. Delaubert, N. Treps, C. Fabre, H. A. Bachor, and P. Réfrégier, Europhys. Lett. 81, 44001 (2008).

[30] C. J. Pethick and H. Smith, Bose-Einstein Condensation in Dilute Gases (Cambridge University Press, Cambridge, 2002).

[31] S. Burger, K. Bongs, S. Dettmer, W. Ertmer, K. Sengstock, A. Sanpera, G. V. Shlyapnikov, and M. Lewenstein, Phys. Rev. Lett. 83, 5198 (1999).

[32] J. Dziarmaga and K. Sacha, Phys. Rev. A 66, 043620 (2002).

[33] K. Mølmer, Phys. Rev. A 58, 566 (1998).

[34] D. S. Petrov, G. V. Shlyapnikov, and J. T. M. Walraven, Phys. Rev. Lett. 85, 3745 (2000).

[35] E. B. Kolomeisky, T. J. Newman, J. P. Straley, and X. Qi, Phys. Rev. Lett. 85, 1146 (2000).

[36] V. A. Brazhnyi, V. V. Konotop, and L. P. Pitaevskii, Phys. Rev. A 73, 053601 (2006).

[37] P. K. Shukla and B. Eliasson, Phys. Rev. Lett. 96, 245001 (2006).

[38] K. W. Madison, F. Chevy, W. Wohlleben, and J. Dalibard, Phys. Rev. Lett. 84, 806 (2000).

[39] L. Salasnich, A. Parola, and L. Reatto, Phys. Rev. A 65, 043614 (2002).

[40] F. Gerbier, Europhys. Lett. 66, 771 (2004).

[41] G. Theocharis, P. G. Kevrekidis, M. K. Oberthaler, and D. J. Frantzeskakis, Phys. Rev. A 76, 045601 (2007).

[42] A. M. Mateo and V. Delgado, Phys. Rev. A 77, 013617 (2008).

[43] K. Huang, Statistical mechanics (John Wiley, New York, 1987).

[44] A. Auerbach, Interacting Electrons and Quantum Magnetism (Springer-Verlag, New York, 1994).

[45] E. V. Shuryak, The QCD vacuum, hadrons and superdense matter (World Scientific, Singapore, 2004).

[46] F. Dalfovo, S. Giorgini, L. P. Pitaevskii, and S. Stringari, Rev. Mod. Phys. 71, 463 (1999).

[47] L. V. Hau, B. D. Busch, C. Liu, Z. Dutton, M. M. Burns, and J. A. Golovchenko, Phys. Rev. A 58, R54 (1998).

[48] I. Bloch, Nature Physics 1, 23 (2005).

[49] E. M. Lifshitz and L. P. Pitaevskii, Statistical Physics, Part 2, Volume 9 (Linacre House, Jordan Hill, Oxford, 2002).

[50] Y. Castin, Coherent atomic matter waves, Lecture Notes of Les Houches Summer School (EDP Sciences and Springer-Verlag, Berlin, 2001).

[51] R. V. Lange, Phys. Rev. 146, 301 (1966).

[52] M. Lewenstein and L. You, Phys. Rev. Lett. 77, 3489 (1996).

[53] Y. Castin and R. Dum, Phys. Rev. A 57, 3008 (1998).

[54] J. Dziarmaga, Phys. Rev. A 70, 063616 (2004).

[55] A. E. Muryshev, H. B. van Linden van den Heuvell, and G. V. Shlyapnikov, Phys. Rev. A 60, R2665 (1999).

[56] T. Busch and J. R. Anglin, Phys. Rev. Lett. 84, 2298 (2000).

[57] T. Busch, Theoretical Studies of Degenerate Inhomogeneous Atomic Gases, Ph.D. Thesis (University of Innsbruck, Innsbruck, 2000). 